\title{
Deciding eventual positivity of polynomials
}

\author{
DAVID HANDELMAN \\ Mathematics Department, University of Ottawa, Ottawa, Ontario K1N $9 B 4$, Canada
}

(Received 1 March 1984 and revised 26 November 1984)

\begin{abstract}
Let $P$ and $f$ be polynomials in several (real) variables, with $P$ having no negative coefficients. We give necessary and sufficient conditions for there to exist a positive integer $n$ with $P^{n} f$ having no negative coefficients; roughly speaking, the conditions involve the behaviour of $f$ as a function on the positive orthant, together with its behaviour on a boundary constructed from the supporting monomials of $P$. This completes a series of results due to Poincaré (1883), Meissner (1911), and Polyà (1927). The former discusses the one variable case, the latter two deal with the situation that the Newton polyhedra of both $P$ and $f$ be, respectively, standard hypercubes, standard simplices.
\end{abstract}

\section{Introduction}

Given a polynomial $\boldsymbol{P}$ (in several variables) with non-negative real coefficients, and a polynomial $f$, we determine necessary and sufficient conditions so that there exists an integer $n$ such that $P^{n} f$ has only non-negative coefficients.

The problem is motivated by the study of very special product type actions of tori on UHF $C^{*}$ algebras ([H1], [HR]). A solution amounts to the determination of the positive cone of the $K_{0}$-group of a crossed product $C^{*}$ algebra arising from the representation of the torus whose character is $P$. It can also be interpreted as a specialized Perron-Frobenius theorem for multiplication operators.

The criteria depend on the Newton diagrams and polyhedra attached to $P$ and $f$, and how the polyhedron of the latter can be fitted inside a blown-up version of the former. To give the reader an idea of the nature of the conditions, we consider the following (easier) problem that was solved in [H1]. Given $f$, decide if there exists a polynomial $Q$ with non-negative coefficients so that $Q f$ itself has no negative coefficients. The solution ([H1; V.6]) can be expressed as follows;

For a $d$-tuple of integers $w=(w(1), w(2), \ldots, w(d))$, define $x^{w}$ to be the monomial $x_{1}^{w(1)} x_{2}^{w(2)} \cdots x_{d}^{w(d)}$ in the variables $x_{1}, x_{2}, \ldots, x_{d}$. Negative exponents are allowed and obviously do not alter the problem (so that from now on, 'polynomial' really means Laurent polynomial). Write $f=\sum \lambda_{w} x^{w}$; set

$$
\log f=\left\{w \in \mathbb{Z}^{d} \mid \lambda_{w} \neq 0\right\}
$$

(this is also called the Newton diagram of $f$ ). Let $\operatorname{cvx} \log f$ denote the convex hull of $\log f$ in $\mathbb{R}^{d}$. Then cvx $\log f$ is the Newton polyhedron of $f$. 
Let $F$ be a (proper) face of $\operatorname{cvx} \log f$. Set

$$
f_{F}=\sum_{w \in F} \lambda_{w} x^{w}
$$

(that is, discard from $f$ all of the terms whose exponent does not belong to $F$ ). Then such a $Q$ exists if and only if:

(*) For every face $F$ (including cvx $\log f$ itself) of cvx $\log f, f_{F}$ is strictly positive as a function on $\left(\mathbb{R}^{d}\right)^{++}=\left\{\left(r_{1}, \ldots, r_{d}\right) \in \mathbb{R}^{d} \mid r_{i}>0\right\}$.

The solution to the question considered in this paper is more complicated, because $\log P$ plays a crucial role. The criterion is similar to (*), in that there is a collection of subsets $\{E\}$ of $\log f$ (which can be determined algorithmically) obtained from $\log P$ and its facial structure, such that $P^{n} f$ has only non-negative coefficients for large $n$, if and only if $f_{E}$ (including $f$ ) are strictly positive as functions on $\left(\mathbb{R}^{d}\right)^{++}$. Each set $E$ is a translate of an iterate of a (relative) face of $\log P$.

We require a number of definitions which reflect the geometric nature of the problem.

Let $P$ be the polynomial $\sum \lambda_{w} x^{w}$ in variables $x_{1}, \ldots, x_{d}$ with non-negative real coefficients. Set $\log P=\left\{w \in \mathbb{Z}^{d} \mid \lambda_{w} \neq 0\right\}$. Let $S, F$ be non-empty subsets of $\log P$, and define cvx $\log P$ as the convex hull of $\log P$ in $\mathbb{R}^{d}$, as before. Observe that the vertices of $\operatorname{cvx} \log P$ belong to $\log P$.

The subset $F$ of $\log P$ is a relative face of $\log P$ if there is a face $K$ of $\operatorname{cvx} \log P$ with $F=K \cap \log P$. An iterate of $F$, written $n F$, is the set of sums taken $n$ at a time of elements of $F$. If $F$ is a relative face of $\log P$ corresponding to $K$, then $n F$ is a relative face of $\log P^{n}(=n \log P$ ) corresponding to $n K$ (recall that the coefficients of $P$ are all non-negative). A translate of $F$ is a subset of $\mathbb{Z}^{d}$ of the form

$$
F+z=\{w+z \mid w \in F\} \quad \text { for some } z \text { in } \mathbb{Z}^{d} .
$$

For the finite subset $S$ of $\mathbb{Z}^{d}$, a stratum of $S$ with respect to the relative face $F$ of $\log P$ is a non-void subset $E$ of $S$ with the following two properties:

(i) There exists a positive integer $n$ and a lattice point $z$ (i.e. $z$ lies in $\mathbb{Z}^{d}$ ) such that $E \subset n F+z$.

(ii) Whenever $E \subset N F+z_{0}$ for some $z_{0}$ in $\mathbb{Z}^{d}$ and some positive integer $N$, it follows that $E=\left(N F+z_{0}\right) \cap S$.

Property (i) says that $E$ is contained in a translate of an iterate of $F$, and property (ii) says that it is maximal (as a subset of $S$ ) with respect to (i). For example, in figure $1, \log P$ consists of all the lattice points in the square with vertices $\{(0,0),(0,3),(3,0),(3,3)\}$, and $S$ is the set of lattice points in the indicated quadrilateral; we determine the strata of $S$ with respect to the relative face $F_{1}=$ $\{(3,0),(3,1),(3,2),(3,3)\}$, and then with respect to $F_{2}=\{(0,3)\}$.

It is clear that $\{(2,3),(2,2),(2,1)\},\{(1,2),(1,1),(1,0)\}$, and $\{(0,1)\}$ are contained in disjoint translates of $F_{1}$, and because they are maximal subsets of $S$ contained in vertical lines, each satisfies (ii).

With respect to $F_{2}$ (or any face consisting of a vertex), the strata are precisely the singleton subsets of $S$. 

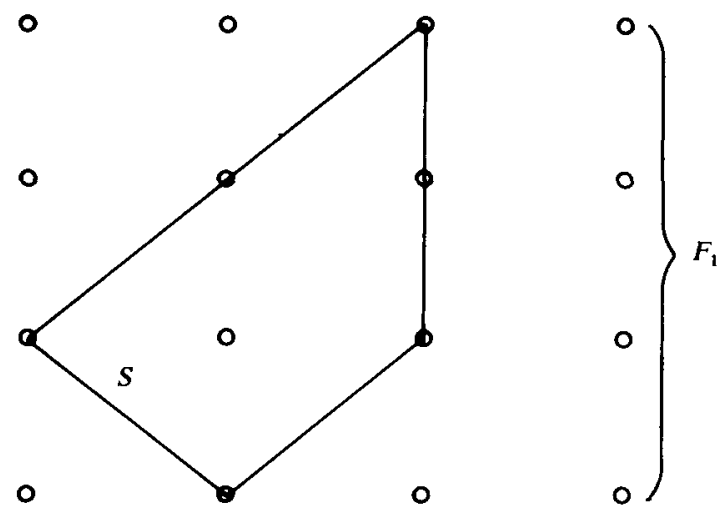

FIGURE 1

In verifying (i) for the subsets of $S$ relative to $F_{1}, n=1$ could be used. An easy example showing that $n>1$ may be required is given in figure 2 . Here $\log P$ is the set of lattice points in the trapezium, $F$ is the relative face at the right, and $S$ is the set of lattice points in the triangle. The strata of $S$ relative to $F$ are $\{(1,0),(1,1),(1,2)\}$ and $\{(0,1)\}$.

o
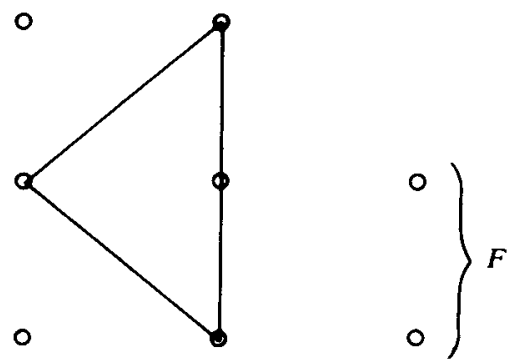

Figure 2

In both cases, for all $k, \log P^{k}=\left(\operatorname{cvx} \log P^{k}\right) \cap \mathbb{Z}^{d}(\log P$ is 'solid'). When this fails, the set of strata can be much more complicated.

LEMMA I.1. Let $F$ be a non-empty relative face of $\log P$, and $S$ a finite subset of $\mathbb{Z}^{d}$. Suppose $n_{1}, n_{2}$ are positive integers, $z_{1}, z_{2}$ are in $\mathbb{Z}^{d}$, and $E_{1}, E_{2}$ are subsets of $S$ satisfying:

$$
E_{1} \subset n_{1} F+z_{1}, \quad E_{2} \subset n_{2} F+z_{2}, \quad E_{1} \cap E_{2} \neq \varnothing .
$$

Then $E_{1} \cup E_{2} \subset n_{3} F+z_{3}$ for some positive integer $n_{3}$ and lattice point $z_{3}$.

In particular, the strata of $S$ relative to $F$ partition $S$, and the intersection of any translate of an iterate of $F$ with $S$ is contained in a unique stratum. 
Proof. Select $e=\left(\sum_{j=1}^{j=n_{1}} v_{j}\right)+z_{1}=\left(\sum_{k=1}^{k=n_{2}} u_{k}\right)+z_{2}$ in $E_{1} \cap E_{2}$, with all the $v_{j}, u_{k}$ lying in $F$. Then

$$
z_{1}-\sum u_{k}=z_{2}-\sum v_{j}=z_{3},
$$

say. We have that $n_{1} F+z_{1} \subset\left(n_{1}+n_{2}\right) F+z_{3}$ (since $z_{1}-z_{3}=\sum u_{k}$, which belongs to $\left.n_{2} F\right)$, and symmetrically $\left(n_{1}+n_{2}\right) F+z_{3} \supset n_{2} F+z_{2}$. With $n_{3}=n_{1}+n_{2}$, the first statement is proved. The comments in the second paragraph are immediate consequences.

Only certain stata are of interest; in some sense, these are the ones closest to $F$. A stratum $E$ of $S$ with respect to $F$ is dominant if it satisfies the following additional condition:

(iii) If $E \subset n \log P+z \backslash(n F+z)$ for some positive integer $n$ and lattice point $z$, then $(n F+z) \cap S=\varnothing$.

For example in figure 1 , with $F_{1}$ as the relative face, only $E=\{(2,1),(2,2),(2,3)\}$ is dominant among the three strata, as a simple argument shows. For $F_{2}=\{(0,3)\}$, there are three dominant strata, the singletons $\{(0,1)\},\{(1,2)\}$, and $\{(2,3)\}$. In figure 2 , only $\{(1,2),(1,1),(1,0)\}$ is a dominant stratum relative to $F$.

There is an algorithm for finding all dominant strata with respect to a given face, which is particularly straightforward if $\log P$ is solid. Select a point $s$ of $S$, and translate an $(y)$ iterate of $F$ so it hits $s$; discard any points of $S$ not in the translate of the iterate of $F$ but which are in the corresponding translate of the iterate of Log $P$. Choose any point of $S$ not already discarded or in a translate of an iterate of $F$ that contains any point previously chosen, and repeat this process until there are no more points of $S$ to choose. What remains is a union of the (disjoint) dominant strata with respect to $F$, and it is routine to separate them.

On a first reading of this paper, it would probably be a good idea to skip the proofs; so the statement of theorem A ( $\$$ II), the statement of theorem B ( $(I V)$, and the examples $(\S \mathrm{V})$ could be read first. Lemma II.4 is a particularly important technical result, used in all parts of the proofs of theorems $\mathrm{A}$ and $\mathrm{B}$.

\section{Statement of the first theorem and a proof of sufficiency}

Let $f=\sum \mu_{w} x^{w}$ be an element of $\mathbb{R}\left[x_{i}^{ \pm 1} ; i=1, \ldots, d\right]$, and $P$ an element of the same ring but having only non-negative coefficients. Let $E$ be a subset of $\log f$; define an element $f_{E}$ of $\mathbb{R}\left[x_{i}^{ \pm 1}\right]$ by throwing away all terms not involving $E$, that is, $f_{E}=\sum_{w \in E} \mu_{w} x^{w}$.

A proper relative face of $\log P$ is a relative face that is not empty and unequal to $\log P$ itself. We often write 'a polynomial has only positive coefficients (or only non-negative coefficients)' as 'a polynomial has no negative coefficients'; this is for variety only. However, the polynomial $f$ is 'strictly positive' (as a function on $\left.\left(\mathbb{R}^{d}\right)^{++}=\left\{r=r\left(r_{1}, \ldots, r_{d}\right) \in \mathbb{R}^{d} \mid r_{i}>0\right\}\right)$ if $f(r)>0$ for all $r$ in $\left(\mathbb{R}^{d}\right)^{++}$.

ThEOREM A. Let $P, f$ be elements of $\mathbb{R}\left[x_{i}^{ \pm 1} ; i \leq d\right]$ with $P$ having only positive coefficients. Then there exists $M$ such that $P^{M} f$ has only positive coefficients if and only if both of the following conditions hold:

(a) Viewed as a function on $\left(\mathbb{R}^{d}\right)^{++}$, for every dominant stratum $E$ with respect to the improper relative face $F=\log P, f_{E}$ is strictly positive. 
(b) For each proper relative face $F$ of $\log P$, and each dominant stratum $E$ of $\log f$ with respect to $F$, there exists $m$ (depending on $F$ and $E$ ) (for which $P_{F}^{m} f_{E}$ has no negative coefficients.

Remarks. (0) If $\log P-\log P$ generates the standard lattice $\mathbb{Z}^{d}$ (inside $\mathbb{R}^{d}$ ) as an abelian group, then of course, (a) becomes:

$f$ is strictly positive on $\left(\mathbb{R}^{d}\right)^{++}$.

More generally, this simplified criterion applies if $\log f$ is contained in $\log P-\log P$ (as we shall see below).

(1) Conditions (a), (b) will be altered to ' $f_{E}$ is strictly positive for all dominant strata $E$ of $\log f$ with respect to the non-empty relative face $F$ of $\log P$, for all (not necessarily proper) faces $F^{\prime}$. This is the content of theorem $\mathrm{B}$, which is proved in $\S \mathrm{IV}$.

(2) After multiplying $P_{F}, f_{E}$ by a suitable monomial, they can be written simultaneously as polynomials involving fewer than $d$ variables (when $F$ is proper). Hence (b) represents a reduction to a (seemingly) less complicated situation.

(3) If $m(E, F)$ is the least integer $m$ such that $P_{F}^{m} f_{E}$ has no negative coefficients, then it will follow from the proof that $M \geq m(E, F)$. On the other hand, (a), (b) do not jointly imply that $P^{M} f_{E}$ has no negative coefficients for any $M$ (example V.6).

The more difficult portion of the argument is the sufficiency of (a) and (b). This will be proved in this section, necessity will be obtained in $\S$ III, and remark 1 (theorem B, the main result of the paper) will be established in $\S$ IV.

We require some definitions, primarily from [H1; $\S \mathrm{I}]$. Here $P=\sum \lambda_{w} x^{w}$ is an element of $A=\mathbb{R}\left[x_{i}^{ \pm 1}\right]$, and we form the ordered ring $S=A\left[P^{-1}\right]$, with ordering given by

$S^{+}=\left\{a P^{-k} \mid a \in A\right.$, there exists $n$ such that $a P^{n}$ has no negative coefficients $\}$. Set

$$
R=\{s \in S \mid \text { there exists a positive integer } N \text { with }-N \cdot 1 \leq s \leq N \cdot 1 \text { in } S\}
$$

( $R$ is the 'bounded subring' of $S$ ). Then $R$ is an ordered ring $\left(R^{+}=S^{+} \cap R\right.$ ), with 1 as an order unit; moreover, $R$ is generated as a real algebra by the set $Z=$ $\left\{x^{w} / P \mid w \in \log P\right\}$, that is, $R=\mathbb{R}\left[x^{w} / P \mid x^{w}\right.$ appears in $\left.P\right]$, and the positive cone of $R$ is generated additively and multiplicatively by $\mathbb{R}^{+}$and $Z$. Thus a typical positive element of $R$ is a positive real-linear combination of terms of the form $\left(x^{w_{1}} / P\right)\left(x^{w_{2}} / P\right) \cdots\left(x^{w_{k}} / P\right)$, where the $w_{i}$ 's all lie in $\log P([\mathrm{H1} ; \S \mathrm{I}])$.

The first step in the proof of sufficiency of (a) and (b), is to show that it is enough to prove it in the case that $\log f \subseteq \log P$. This will occupy the next ten paragraphs.

The first step in this reduction is to multiply $P$ by a monomial (if necessary) so that $0=(0, \ldots, 0)$ belongs to $\log P$. Let $H$ denote the subgroup of the standard lattice, $\mathbb{Z}^{d}=\left\{\left(a_{1}, \ldots, a_{d}\right) \in \mathbb{R}^{d} \mid a_{i} \in \mathbb{Z}\right\}$, generated by $\log P$. Let $\left\{e_{c}\right\}_{c \in C}$ be a transversal of $H$ in the standard lattice, that is $\cup\left(H+e_{c}\right)=\mathbb{Z}^{d}$; let 0 be one of the $e_{c}$ 's, say $0=e_{0}$. For each index $c$, define $p_{c}: \mathbb{R}\left[x_{i}^{ \pm 1}\right] \rightarrow \mathbb{R}\left[x_{i}^{ \pm 1}\right]$ via

$$
p_{c}\left(x^{w}\right)= \begin{cases}0 & \text { if } w \notin H+e_{c}, \\ x^{w} & \text { if } x \in H+e_{c}\end{cases}
$$


extended $\mathbb{R}$-linearly. We observe that for a polynomial $k, k$ belongs to the image of $p_{c}$ if and only if $\log k \subseteq H+e_{c}$. Moreover, $p_{c}(k)=0$ for all but finitely many $c$ 's. Finally, $k$ has no negative coefficients if and only if the same is true of all the $p_{c}(k)$.

Since $\log P^{n} \subseteq H$ (because of our assumption that 0 belongs to $\log P$ ) for all $n$, $p_{c}\left(P^{n} f\right)=P^{n} p_{c}(f)$ for all $c$. Hence $P^{n} f$ has no negative coefficients if and only if the same is true of $P^{n}\left(x^{-e_{c}} p_{c}(f)\right.$ ) (for all $c$ ); of course $\log \left(x^{-e_{c}} p_{c}(f)\right) \subseteq H$. Hence if we can solve the problem of eventual positivity of those $f$ 's with $\log f \subset H$, we can solve the general case (by considering the finite set of polynomials, $\left.\left\{x^{-e_{c}} p_{c}(f) \mid p_{c}(f) \neq 0\right\}\right)$.

Suppose $S$ is a finite subset of $H$; we show there exists $v$ in $H$ and a positive integer $m$ with $S+v \subseteq \log P^{m}$ (the idea is to apply this to $S=\log \left(x^{-e_{c}} p_{c}(f)\right)$ ). For each $s$ in $S$, we may write $s=\sum \mu_{s w} w$ with $w \in \log P, \mu_{s w} \in \mathbb{Z}$, since $H$ is generated by $\log P$. Form $J_{s}=\left\{(s, w) \mid \mu_{s w}<0\right\}$, and set $z_{s}=\sum_{J_{s}}\left(-\mu_{s w}\right) w$. Set $N_{s}=\max \left|\mu_{s w}\right|$; then $s+z_{s}$ belongs to $N_{s} \log P$, and moreover, $z_{s}$ also so belongs. Set $v=\sum_{s} z_{s}$ and $m=\sum_{S} N_{s}$, and we are done.

With $S=\bigcup \log \left(x^{-e}{ }_{c} p_{c}(f)\right) \subset H$, we may thus find a monomial $x^{v}$ so that $\log \left(x^{v} x^{-e_{c}} p_{c}(f)\right) \subseteq \log P^{m}$. Now we must show that this reduction (to all of the $p_{c}(f)$ 's) actually preserves the notions of strata and dominant strata.

First, we show that if $F$ is a relative face of $\log P$, then $n F=\left\{\sum^{n} v_{i} \mid v_{i} \in F\right\}$ is a relative face of $\log P^{n}=n \log P$. This follows from [H; III.1b] and the fact that if $\left\{w_{i} \mid i=1, \ldots, n\right\}$ is a subset of $\log P$ and $\sum w_{i}$ belongs to the convex hull of $n F$, then each $w_{i}$ belongs to the convex hull of $F$; however, $F=\operatorname{cvx} F \cap \log P$, so $w_{i}$ belongs to $F$, and thus $\sum w_{i}$ lies in $n F$.

Now suppose that $E$, a subset of $S$, is a (dominant) stratum (of $S$ ) with respect to $F$, a relative face of $\log P$. Then certainly $E$ is a (dominant) stratum of $S$ with respect to the relative face $n F$ of $\log P^{n}$, just from the definitions. Conversely, suppose that $E \subset S$ is a (dominant) stratum with respect to $n F$. We wish to show that it is a (dominant) stratum with respect to $F$.

To see that $E$ is at least a stratum, we see that

$$
E \subset m_{0}(n F)+z_{0}=m_{0} n F+z_{0},
$$

for some positive integer $m_{0}$ and lattice point $z_{0}$. Suppose that $E \subset m F+z$ ( $m$ a positive integer, $z$ a lattice point), and $w$ belongs to $S \cap(m F+z)$. There exists a positive integer $k$ so that $m<k n$. Select $v$ in $(k n-m) F$. Then $m F+z \subset k n F+(z-v)$. Thus,

$$
E \subset k(n F)+(z-v) \text { and } w \in S \cap(k(n F)+(z-v)) .
$$

Since $E$ is a stratum of $S$ with respect to $n F$, this implies $w$ belongs to $E$, so that $E=S \cap(m F+z)$, and thus $E$ is a stratum with respect to $F$.

Suppose $E$ is dominant with respect to $n F$ (as a subset of $S$ ); we show it is dominant with respect to $F$. If $E \subset(m \log P+z) \backslash(m F+z)$ (for a positive integer $m$ and lattice point $z)$, we require that $(m F+z) \cap S=\varnothing$. Choose $k$ a positive integer so that $k n>m$, and select $v$ in $(k n-m) F$. In particular, $v$ lies in $(k n-m) \log P$ and $E \subset k n \log P+(z-v)$. If $e$ lies in $E \cap(k n F+z-v)$, we may write $e=h+z-v$ 
with $h$ in $k n F$, so $h=(e-z)+v$. Now $e-z$ belongs to $m \log P$ (from $E \subset m \log P+$ $z$ ), and $v$ lies in $(k n-m) F$, so from the facial property of $F, e-z$ is a member of $m F$; this means $e$ belongs to $m F+z$, a contradiction. Hence $E \cap(k n F+z-v)=\varnothing$. So we have

$$
E \subset(k(n \log P)+(z-v)) \backslash(k(n F)+(z-v)) .
$$

From the definition of dominant stratum (with respect to $n F),(k(n F)+(z-v)) \cap S=$ $\varnothing$. If $s \in m F+z$, then $s \in k n F+(z-v)$, whence $(m F+z) \cap S=\varnothing$, as desired.

Next we observe that if $E$ is a stratum of $S$ with respect to a relative face $F$ of $\log P$, then $E \subset H+e_{c}$ for some $e_{c}$ : there exists an integer $m$ and a lattice point $z$ so that $E \subset m F+z \subset m \log P+z \subset H+z$, one of the cosets. Hence $f_{E}=p_{c}(f)$ when $F=\log P$. On the other hand, if $E$ is a non-empty dominant stratum of $S \cap\left(H+e_{c}\right)$ (for some $c$ ) with respect to $F$, then it is also a dominant stratum of $S$. This follows directly from the definitions.

To summarize: begin with $f$ and $P$. There exists $n$ such that $P^{n} f$ has no negative coefficients if and only if the same is true of $P^{n} p_{c}(f)$ for all $c$ such that $p_{c}(f) \neq 0$. The dominant strata of $\log f(=S)$ with respect to a relative face $F$ of $\log P$ each lie entirely within one coset of $H$. We thus deduce that every $p_{c}(f)$ satisfies the hypotheses in the theorem(s) on its dominant strata if and only if $f$ does. Moreover, replacing $p_{c}(f)$ by $g_{c}=x^{-e_{c}} p_{c}(f)$, we have only to verify that the theorems hold for $\log f \subset H$. By multiplying $g_{c}$ by a suitable monomial, we may assume that $\log f \subset$ $\log P^{N}$ for some $N$. Dominant strata are the same with respect to $F$ as $N F$, so we may replace $P$ by $P^{N}$. We thus reduce our problem to the case that $\log f \subset \log P$.

Assume that $\log f \subset \log P$, and let $I$ denote the order-ideal of $R$ generated by $B=\left\{x^{v} / P \mid v \in \log f\right\}$. Then $I$ is actually the $R$-ideal generated by the elements of $B$. To see this observe that if $0 \leq s \leq N\left(\sum_{B} x^{v} / P\right)$, then there exist $s_{v}$ in $R$ such that $0 \leq s_{v} \leq N x^{v} / P$ for each element of $B$ (by Riesz interpolation [H1; $\S 1$ ), and $0 \leq$ $s_{v} P x^{-v} \leq N$ in $S$, so $s_{v}$ belongs to the $R$-ideal generated by $\left\{x^{v} / P\right\}$.

Define $\log I=\left\{v \in \log P \mid x^{v} / P \in I\right\}$. Clearly $\log f \subset \log I$, although this inclusion may be strict. It happens to be technically easier to deal with Log $I$. We must show that there is a strong connection between the dominant strata of Log $I$ and of $\log f$.

LeMMA II.1. Let $\mathrm{S} \subset \log P, F$ a relative face of $\log P, E$ a dominant stratum of $S$ with respect to $F$, and $N$ a positive integer. Suppose that $w$ lies in $(N \log P+S) \cap$ $(N F+E)$. Then whenever $w$ can be expressed as $w=s+t$ with $s$ in $N \log P$ and $t$ in $S$, we have that $t$ belongs to $E$.

Proof. There exists a positive integer $n$ and a lattice point $z_{0}$ so that $E \subset n F+z_{0}$. We may write $s+t=q+e$ with $q$ in $N F$ and $e$ in $E$. Thus

$$
t=q+e-s \in N F+E-s \in(N+n) F+\left(z_{0}-s\right) .
$$

Suppose that $t$ does not belong to $E$. Since $t$ does lie in $S$, we have that $E \cap$ $\left\{(N+n) F+\left(z_{0}-s\right)\right\}=\varnothing$ (let $V$ denote the intersection; then $V \cup\{t\}$ is contained in $\left((N+n) F+\left(z_{0}-s\right)\right) \cap S$, so that $V \cup\{t\}$ is contained in a stratum; if $V$ is non-empty, the stratum must be $E$; thus $t$ would belong to $E$, a contradiction). 
Since $E \subset n \log P+z_{0}$ and $s$ belongs to $N \log P$, it follows that

$$
E \subset\left((N+n) \log P+\left(z_{0}-s\right)\right) \backslash\left((N+n) F+\left(z_{0}-s\right)\right),
$$

and

$$
t \in(N+n) F+\left(z_{0}-s\right) .
$$

This contradicts (iii) of the definition of dominant stratum.

Lemma II.2. Let $S_{0} \subset S \subset \log P$, and $F$ a relative face of $\log P$.

(a) If $E$ is a stratum of $S$ (with respect to $F$ ) and $E \cap S_{0} \neq \varnothing$, then $E \cap S_{0}$ is a stratum of $S_{0}$ with respect to $F$.

(b) Given a stratum $D$ of $S_{0}$, there exists a stratum $E$ of $S$ such that $D=E \cap S_{0}$.

Proof. (a) Let $E$ be a stratum of $S$ with $D=E \cap S_{0} \neq \varnothing$. Then $D$ is contained in a translate of an iterate of $F$, and thus by $I .1, D$ is contained in a stratum of $S_{0}$, say $E^{\prime}$. But $E^{\prime} \cap E \neq \varnothing$, so again by I.1, $E^{\prime} \cup E$ is contained in a stratum of $S$. Thus $E=E^{\prime} \cup E$, so $E^{\prime} \subset E$. It follows that $D=E^{\prime}$, and therefore $D$ is a stratum of $S_{0}$.

(b) Since $D$ is contained in a translate of an iterate of $F, D$ is contained in a stratum, $E$, of $S$. Hence $D \subset E \cap S_{0}$; since the latter is contained in a translate of an iterate of $F$ and $D$ is a stratum of $S_{0}, D=E \cap S_{0}$.

Proposition II.3. If $E$ is a dominant stratum of $\log I$ with respect to a relative face $F$ of $\log P$, then $E \cap \log f$ is not empty, and thus is a stratum of $\log f$.

Proof. We show that $E \cap \log f \neq \varnothing$, so that II.2(a) applies. Choose $e$ in $E$. Since $x^{e} / P$ is in the order ideal generated by $B\left(=\left\{x^{v} / P \mid v \in \log f\right\}\right)$, we have that for some integer $N$,

$$
x^{e} / P \leq N\left(\sum_{B} x^{v} / P\right) \quad \text { in } R .
$$

Hence there is a positive integer $\mathrm{K}$ so that for every monomial $x^{w}$, the coefficient of $x^{w}$ in $x^{e} P^{K}$ is dominated by the coefficient of $x^{w}$ in $N\left(\sum_{B} x^{v} P^{K}\right)$. Thus

$$
e+K \log P \subset \log f+K \log P \text {. }
$$

Since there are only finitely many $e$ 's in $E$, by suitably increasing $K$, we have that $E+K \log P$ is contained in $\log f+K \log P$. Thus

$$
E+K F \subset \log f+K \log P \subset \log I+K \log P .
$$

Choose $w$ in $F$, and consider $z=e+K w \in \log I+K \log P$, for any $e$ in $E$. Obviously $z$ belongs to $E+K F$, so by the inclusion above, there exist $v$ in $\log f$ and $s$ in $K \log P$ such that $z=v+s$. By II.1, $v$ belongs to $E$, so $\log f \cap E$ is not empty.

It is not clear (at the time of writing) that if $D$ is a dominant stratum of $\log f$ and $D=E \cap \log f$ for $E$ a stratum of $\log I$, that $E$ is dominant. Oddly enough, this is irrelevant (although it appears to be true).

To show that $f$ is a positive element of $S$, we must show that $f / P$ is a positive element of $R$ (since $\log f \subset \log P, f / P$ belongs to $R$ in any case). By construction, $f / P$ belongs to $I$. As $I$ is generated as order-ideal by the finite set $B, I$ admits an order unit, e.g. $\sum_{w \in \log f} x^{w} / P$ (in fact, since $R$ is noetherian, all order-ideals of $R$ 
admit order units). We show that for all pure states $\theta$, of $I, \theta(f / P)>0$. By [EHS; I.4], $f / P$ will then be a positive element of $R$, and we will be done.

By [H1; I.2], the pure states of $I$ are of two distinct forms. Either $\theta$ is a pure state of $R$, hence is multiplicative (possibly after a renormalization), or there exists a pure state $L$ of $R$ with $L(I)=0$ such that for $i$ in $I$ and $r$ in $R, \theta(i r)=\theta(i) L(r)$.

By replacing $P$ by a sufficiently high power of itself if necessary and then translating $f$ (replacing $f$ by $x^{w} f$ for some suitable $w$ ), we may assume that $\log f$ is disjoint from all the proper relative faces $F$ of $\log P$. Since $\left\{x^{w} / P \mid w \in \log P \backslash F\right\}$ generates a proper order ideal $J$ such that $x^{w} / P \in J$ (for $w$ in Log $P$ ) implies $w \notin F$ [H1; VII], it follows that $\log I \cap F=\varnothing$ for all relative faces $F$.

Now we can dispose of the pure states arising as restrictions of pure states of $R$. Let $\theta$ be such a state; set $K=\left\{w \in \log P \mid \theta\left(x^{w} / P\right) \neq 0\right\}$. Either $K$ is all of $\log P$, or $K$ is a proper relative face of $\log P$ [H1; III.2]. In the latter case, $\theta$ kills $I$, so does not restrict to a state. In the former situation, $\theta$ arises from an evaluation at a point of $\left(\mathbb{R}^{d}\right)^{++}$, i.e. $\theta$ is an evaluation at $r=\left(r_{1}, \ldots, r_{d}\right)[H 1 ; 1.3 \&$ II.5]. Then $\theta(f / P)=f(r) / P(r)>0$, as desired. The rest of this section is devoted to the case that arises from a pure state of $R, L$, such that $L(I)=0$, as mentioned above.

Let $\theta$ be such a state, and $L$ its corresponding pure state on $R$. Then $F=$ $\left\{w \in \log P \mid \theta\left(x^{w} / P\right) \neq 0\right\}$ is a relative face of $\log P$ (affiliated to $\theta$ ), and the ideal ( $=$ order-ideal) generated by $\left\{x^{w} / P \mid w \in \log P \backslash F\right\}$ is prime and generated by $\operatorname{Ker} L \cap R^{+}([\mathrm{H1} ; \S \mathrm{VII}])$.

Let $E$ be a subset of $\log I$ with the following properties:

(a) for all $e$ in $E, \theta\left(x^{e} / P\right) \neq 0$;

(b) for some positive integer $k$, some lattice point $z, E \subset k F+z$;

(c) whenever $E \subset m F+z_{0}$, then

$$
\begin{aligned}
E & =\log I \cap(m F+z) \cap\left\{w \in \log I \mid \theta\left(x^{w} / P\right) \neq 0\right\} \\
& =\left\{w \in \log I \mid\left(x^{w} / P\right) \neq 0\right\} \cap\left(m F+z_{0}\right) .
\end{aligned}
$$

So $E$ is almost a stratum; we will show in fact that $E$ is a dominant stratum, and equals $E_{0}=\left\{w \in \log I \mid \theta\left(x^{w} / P\right) \neq 0\right\}$.

To this end, we define a sequence of functions $\Xi_{k}, k=1,2, \ldots$, as follows. For $a$ in $\mathbb{R}\left[x_{i}^{ \pm 1}\right]$ with $\log a \subset \log I+(k-1) \log P$, set $\Xi_{k}(a)=a_{E+(k-1) F}$. We have a candidate mapping $\psi: I \rightarrow \mathbb{R}$, trying to send $a / P^{k}$ to $\theta\left(\Xi_{k}(a) / P^{k}\right)$. We now show that this is well defined and a state of $I$.

Proof that $\psi$ is well defined. We shall show that $\psi$ is independent of the choice of representatives (of $a / P^{k}$ ) and is defined on all of $I$.

Define $\log _{k} I=\log I+k \log P$. An essential technical result is the following: $E+j F$ satisfies properties (a), (b), (c) above (which $E$ satisfies with respect to $\log I$ ) with respect to $\log _{j} I$, for $j=1,2, \ldots$.

(a) If $v=w+\sum w_{i}$, with $w$ in $E$, the $w_{i}$ 's in $F$, then

$$
\theta\left(x^{v} / P^{j+1}\right)=\theta\left(x^{w} / P\right) \cdot\left(\prod L\left(x^{w_{i}} / P\right)\right)
$$

As the $w_{i}$ 's belong to $F$, the values of $L$ are all non-zero verifying (a).

(b) Clearly,

$$
E+j F \subset(\log I+j \log P) \cap((j+k) F+z)
$$


(c) Suppose that $E+j F \subset(\log I+j \log P) \cap\left(t F+z_{0}\right)$, and let $y$ belong to the right side, and suppose that $\theta\left(x^{y} / P^{j+1}\right) \neq 0$. Write $y=w+\sum_{1}^{j} w_{i}$, with $w$ in $\log I$ and the $w_{i}$ in $\log P$. If any of the $w_{i}$ did not belong to $F$, then $L\left(x^{w_{i}} / P\right)$ would be zero, and thus so would be $\theta\left(x^{y} / P^{j+1}\right)$. Hence each of the $w_{i}$ belongs to $F$, and also $\theta\left(x^{w} / P\right) \neq 0$.

Now write $y=z_{0}+\sum v_{m}$, with the $v_{m}$ 's in $F(m=1,2, \ldots, t)$. We obtain that $w=\sum v_{m}-\sum x_{i}+z_{0} \in t F+\left(z_{0}-\sum w_{i}\right)$. From $E+j F \subset t F+z_{0}, E+\sum w_{i} \subset t F+z_{0}$ follows; thus $E \subset t F+\left(z_{0}-\sum w_{i}\right)$. By property (c) for $E, w$ belongs to $E$, and so $y$ belongs to $E+j F$ as desired.

Now we show that $\psi$ is defined on all of $I$ (but not necessarily well defined). Suppose $a / P^{k}$ belongs to $I$; we require that there exist an integer $n$ such that $\log a P^{n} \subset \log _{n+k-1} I$ (for then $\psi$ would be defined on $a P^{n} / P^{n+k}$ ).

First we prove that for each positive integer $t$, there exists an integer $n$ such that $\log _{n} I \supset\left\{w \in t \log P \mid x^{w} / P^{t} \in I\right\}$. Let $u=\sum_{v \in \log I} x^{v} / P$; then $u$ is an order unit for $I$. Setting $a_{w}=x^{w} / P^{t}$ for $w \in t \log P$ and $a_{w} \in I$, there exists an integer $N$ such that $a_{w} \leq N u$. By interpolation, there exist $0 \leq b_{v} \leq N x^{v} / P$ with $a_{w}=\sum_{v} b_{v}$, and as before II.1, there exist $r_{v}$ in $R^{+}$with $b_{v}=\left(x^{v} / P\right) r_{v}$. There exists $m$ so that for all $v$, we may write $r_{v}=c_{v} / P^{m}$, where $c_{v}$ are in $\mathbb{R}\left[x_{i}^{ \pm 1}\right]$ and have no negative coefficients. Set $n=m+1$; the desired inclusion then follows.

Given $r=a / P^{k}$ in $I$, we may write $r=r_{1}-r_{2}$ with $r_{1}, r_{2}$ in $I^{+}$; we may thus write $r_{j}=a_{j} / P^{m}, j=1,2$, with $a_{j}$ in $\mathbb{R}\left[x_{i}^{ \pm 1}\right]$ having no negative coefficients. Each term $x^{w} / P^{m}$ appearing in $a_{j} / P^{m}$ belongs to $I$, by the convexity of $I$. Hence there exists $I$ so that $\log P^{m} a \subset \log _{m+k-1} I$. The upshot of this is that the candidate function $\psi$ is at least defined on all of $I$. Now we show that it is well defined.

Suppose that $a, b$ are Laurent polynomials, with $a / P^{k}=b / P^{m}$ in $I$, and moreover that $m>k$ and $\log a \subset \log _{k-1} I, \log b \subset \log _{m-1} I$. We wish to show that

Write

$$
\theta\left(a_{E+(k-1) F} / P^{k}\right)=\theta\left(b_{E+(m-1) F} / P^{m}\right) \text {. }
$$

Since $b=P^{m-k} a$, we have

$$
b=\sum \rho_{w} x^{w}, \quad a=\sum \mu_{v} x^{v}, \quad P^{m-k}=\sum_{s \in(m-k) \log P} \lambda_{s} x^{s} .
$$

$$
\sum \rho_{w} x^{w}=\sum_{w}\left(\sum_{\substack{s+v=w \\ s \in(m-k) \log P \\ v \in \log _{k-1} I}} \lambda_{s} \mu_{v}\right) x^{w} .
$$

By definition,

On the other hand,

$$
b_{E+(m-1) F}=\sum_{w \in E+(m-1) F}\left(\sum_{\substack{s+v=w \\ s \in \log ^{m-k} \\ v \in \log _{k-1} I}} \lambda_{s} \mu_{v}\right) x^{w} .
$$

$$
\begin{aligned}
P^{m-k} a_{E+(k-1) F} & =\left(\sum_{(m-k) \log P} \lambda_{s} x^{s}\right) \cdot\left(\sum_{E+(k-1) F} \mu_{v} x^{v}\right) \\
& =\sum_{w \in((m-k) \log P)+E+(k-1) F}\left(\sum_{\substack{s+v=w \\
s \in(m-k) \log P \\
v \in E+(k-1) F}} \lambda_{s} \mu_{v}\right) x^{w} .
\end{aligned}
$$


Now we remark that if $w$ belongs to $(m-k) \log P+E+(k-1) F$, but not to $E+(m-1) F$, then $\theta\left(x^{w} / P\right)=0$. Write $w=\sum w_{i}+e+\sum g_{j}$, with $w_{i}$ in $\log P, g_{j}$ in $F$, and at least one of the $w_{i}$ 's not in $F$. Then for $w_{i} \notin F, L\left(x^{w_{i}} / P\right)=0$, so $\theta\left(x^{w} / P^{m}\right)=0$. Similarly, if $s+v=w, s$ belongs to $(m-k) \log P, v$ lies in $E+(k-1) F$, and $s$ does not belong to $(m-k) F$, then $\theta\left(x^{w} / P^{m}\right)=0$. Combining this with (2) gives

$$
\theta\left(\frac{P^{m-k} a_{E+(k-1) F}}{P^{m}}\right)=\theta\left\{\sum_{w \in(m-1) F+E}\left(\sum_{\substack{s+v=w \\ s \in(m-k) F \\ v \in E+(k-1) F}} \lambda_{s} \mu_{v}\right)\left(x^{w} / P^{m}\right)\right\} .
$$

By definition,

$$
\theta\left(\frac{b_{E+(m-1) f}}{P^{m}}\right)=\theta\left\{\sum_{w \in E+(m-1) F}\left(\sum_{\substack{s+v=w \\ s \in(m-k) \log P \\ v \in \log I+(k-1) \log P}} \lambda_{s} \mu_{v}\right)\left(x^{w} / P\right)\right\} .
$$

Now suppose $w_{0}$ belongs to $E+(m-1) F$, and $w_{0}=s+v$, where $s$ lies in $(m-k) \log P$ and $v$ in $\log I+(k-1) \log P$. If we assume that $\theta\left(x^{w_{0}} / P\right)$ is not zero (for otherwise, it does not contribute to the right side of (4)), then we obtain, as before, that $s$ belongs to $(m-k) F$ and $v$ belongs to $\log I+(k-1) F$. We will show that $v$ must lie in $E+(k-1) F$, so that the terms on the left sides of (3) and of (4) are equal.

There exists an integer $n$ and a lattice point $z_{0}$ with $E+(k-1) F \subset n F+z_{0}$; from $s+v$ lying in $E+(m-1) F$, we obtain tht $s+v$ belongs to $E+(k-1) F+(m-k) F \subset$ $(n+m-k) F+z_{0}$. Hence $v$ is a member of $(m+n-k) F+\left(z_{0}-s\right)$. However, $s$ belongs to $(m-k) F$ and $E+(k-1) F \subset n F+z_{0}$, so $E+(k-1) F \subset(n+m-k) F+$ $\left(z_{0}-s\right)$. Since $\theta\left(x^{v} / P^{k}\right)$ is not zero (else $\left.\theta\left(x^{w_{0}} / P^{m}\right)=0\right)$, and $v$ lies in Log $I$, we have by property (c) with $E+(k-1) F$, that $v$ belongs to $E+(k-1) F$.

Hence $\psi$ is independent of the representation of $a / P^{k}$, and is thus well defined.

Proof that $\psi$ is a state of $I$. We check that $\psi$ is additive to begin with. Choose $a, c$ in $\mathbb{R}\left[x_{i}^{ \pm 1}\right]$ with $\log a \subset \log _{k-1} I, \log c \subset \log _{m-1} I$ (so $a / P^{k}, c / P^{m}$ both belong to $I$ ), and suppose that $m \geq k$. Then

$$
\begin{aligned}
\psi\left(\left(a / P^{k}\right)+\left(c / P^{m}\right)\right) & =\psi\left(\frac{P^{m-k} a+c}{P^{m}}\right) \\
& =\theta\left(\frac{\Xi_{m}\left(P^{m-k} a+c\right.}{P^{m}}\right) \\
& =\theta\left(\frac{\left(P^{m-k} a+c\right)_{E+(m-1) F}}{P^{m}}\right) \\
& =\theta\left(\frac{\left(P^{m-k} a\right)_{E+(m-1) F}+c_{E+(m-1) F}}{P^{m}}\right) \\
& =\theta\left(\frac{\left(P^{m-k} a\right)_{E+(m-1) F}}{P^{m}}\right)+\theta\left(\frac{c_{E+(m-1) F}}{P^{m}}\right) \\
& =\psi\left(a / P^{k}\right)+\psi\left(c / P^{m}\right) .
\end{aligned}
$$

Now suppose that $a / P^{k}$ is positive in $R$ and belongs to $I$. There exists an integer 
$n$ so that $P^{n} a$ has only non-negative coefficients. Then

$$
\psi\left(a / P^{k}\right)=\psi\left(P^{n} a / P^{n+k}\right)=\theta\left(\left(P^{n} a\right)_{E+(n+k-1) F} / P^{n+k}\right),
$$

and the latter is non-negative since $\theta$ is a state. Thus $\psi$ is a state of $I$.

Recall that $E_{0}=\left\{w \in \log I \mid \theta\left(x^{w} / P\right) \neq 0\right\}$. Pick $e$ in $E_{0}$ and $z$ in $F$. Embed $e$ in a stratum of $\log I$ with respect to $F$, call it $E_{1}$. Then $E=E_{0} \cap E_{1}$ satisfies (a) and (b) above. We show that it also satisfies (c).

If $E \subset m F+z_{0}$, then $e$ belongs to $m F+z_{0}$, so $\log I \cap\left(m F+z_{0}\right)$ is contained in the stratum $E_{1}$ of $\log . I$. Thus $\left(\log I \cap\left(m F+z_{0}\right)\right) \cap E_{0}$ is contained in $E_{1}$, and so in $E$. The reverse inclusion is obvious, so (c) holds.

With the state $\psi: I \rightarrow \mathbb{R}$ defined as above with this choice of $E, \psi(r) \leq \theta(r)$ for all $r$ in $I^{+}$, so $\psi \leq \theta$ as states of $I$. Since $\theta$ is pure, there exists a real number $\lambda$ such that $\psi=\lambda \theta$. As $E$ is not empty, $\psi$ is not zero, so $\lambda>0$; moreover, for $w$ in $E$, $\psi\left(x^{w} / P\right)=\theta\left(x^{w} / P\right) \neq 0$, so $\lambda=1$. Hence $\psi=\theta$. For $w$ in $E_{0}, \theta\left(x^{w} / P\right)=\psi\left(x^{w} / P\right) \neq 0$, so $w$ belongs to $E$, whereby $E=E_{0}$.

Now we show that $E_{0}$ is a stratum. Suppose $E_{0} \subset n F+z$; it is required to prove that $E_{0}=(n F+z) \cap \log I$. If the right side is a singleton, equality holds, as $\theta$ is not identically zero. Otherwise, select $e$ in $E_{0}$ and $e^{\prime}$ in the right side unequal to $e$. Write $e^{\prime}=w+z, e=w^{\prime}+z$, where $w, w^{\prime}$ belong to $n F$. Then $e+w=e^{\prime}+w^{\prime}$, so

$$
\theta\left(\left(x^{e} / P\right) \cdot\left(x^{w} / P^{n}\right)\right)=\theta\left(\left(x^{e^{\prime}} / P\right) \cdot\left(x^{w^{\prime}} / P^{n}\right)\right) .
$$

The leftmost term is $\theta\left(x^{e} / P\right) L\left(x^{w} / P^{n}\right)$, which is not zero since $w$ belongs to $n F$. The right term is $\left.\theta\left(x^{e^{\prime}}\right) / P\right) L\left(x^{w^{\prime}} / P^{n}\right)$; hence $\theta\left(x^{e^{\prime}} / P\right)$ is not zero, so $e^{\prime}$ belongs to $E_{0}$. Thus $E_{0}=(n F+z) \cap \log I$, and so $E_{0}$ is a stratum.

Finally, we show that $E_{0}$ is a dominant stratum of $\log I$, with respect to $F$. To see this, suppose that $w$ is an element of $\log I \cap(n F+z)$ and $E_{0} \subset$ ( $n \log P+z) \backslash(n F+z)$. Write $w=\sum_{1}^{n} v_{j}+z$, where the $v_{j}$ 's belong to $F$; select any $e$ in $E_{0}$, and write it $e=\sum p_{j}+z$, with the $p_{j}$ 's in $\log P$. At least one of the $p_{j}$ 's does not lie in $F$.

From $w+\sum p_{j}=e+\sum v_{j}$, we deduce

$$
\theta\left(\frac{x^{w}}{P} \cdot \prod_{j} \frac{x^{p_{j}}}{P}\right)=\theta\left(\frac{x^{e}}{P} \cdot \prod_{j} \frac{x^{v_{j}}}{P}\right)
$$

The left side is a product involving $L\left(x^{p_{j}} / P\right)$, so is zero. On the other hand the right side is $\theta\left(x^{e} / P\right) \cdot \prod L\left(x^{v_{j}} / P\right)$ which is not zero since $e$ belongs to $E_{0}$ and $v_{j}$ to $F$. This contradicts the existence of $w$, so $E_{0}$ is dominant.

LEMMA II.3 A. If $\theta$ is a pure state of $I$ with corresponding relative face $F$ and pure state $L$ of $(R, 1)$, then the set

$$
E=\left\{e \in \log f \mid \theta\left(x^{e} / P\right) \neq 0\right\}
$$

is a dominant stratum of $\log f$ with respect to $F$.

Proof. We have seen that $E_{0}=\left\{w \in \log I \mid \theta\left(x^{w} / P\right) \neq 0\right\}$ is a dominant stratum of $\log I$, and $E_{0} \cap \log f=E$, so $E$ is a stratum of $\log f$ by II.2 and II.3.

Suppose that $E \subset(n \log P+z) \backslash(n F+z)$. for some integer $n$ and lattice point $z$, and suppose that there exists $w$ in $\log f \cap(n F+z)$. Select $e$ in $E$ and write 
$e=\sum w_{i}+z$, with the $w_{i}$ in $\log P$, but not all of them in $F$, and $w=\sum v_{i}+z$ with the $v_{i}$ in $F$. Then $e+\sum v_{i}=w+\sum w_{i}$, so applying $\theta$ to

$$
\left(\frac{x^{e}}{P}\right) \cdot \prod\left(\frac{x^{v_{i}}}{P}\right)=\left(\frac{x^{w}}{P}\right) \cdot \prod\left(\frac{x^{w_{i}}}{P}\right)
$$

makes the left side $\theta\left(x^{e} / P\right) \cdot L\left(x^{v_{i}} / P\right)$. This is not zero, since all of the $v_{i}$ 's belong to $F$. However, not all of the $w_{i}$ 's lie in $F$, so that $\theta$ of the right hand side is zero, a contradiction.

LEMMA II.4 (conclusion to II.1). With the notation and hypotheses of II.1, whenever $w$ in $(N \log P+S) \cap(N F+E)$ can be expressed as $w=s+t$, where $s$ belongs to $N \log P$ and $t$ to $S$, it follows that $t$ belongs to $E$ and $s$ belongs to NF.

Proof. That $t$ lies in $E$ is the conclusion of II.1. From $E \subset n F+z_{0}$ and $w$ in $N F+E$, we have that $w$ belongs to $(N+n) F+z_{0}$. Thus $s+t-z_{0}$ is a member of $(N+n) F$. Since $t$ lies in $E$ (which is contained in $n F+z_{0}$ ), we have that $t-z_{0}$ belongs to $n F$, which is in turn contained in $n \log P$.

Set $d=x^{s} / P^{N}, g=x^{t-z_{0}} / P^{n}$. Both are elements of $R$ (and thus of $R^{+}$) by the preceding paragraph. Let $L$ be any pure state of $R$ with the property that $L\left(x^{v} / P\right)=0$ (for $v$ in $\log P$ ) if and only if $v$ does not lie $F$. If $s$ does not belong to $N F$, then $d$ is in the kernel of $L$, so that so is $d g$. But this entails $s+t-z_{0}$ not belonging to $(N+n) F$, a contradiction.

We can now prove the sufficiency portion of theorem A. There exists (by hypothesis) an integer $m$ so that for any dominant stratum $E$ of $\log f,\left(P_{F}\right)^{m} f_{E}$ has only non-negative coefficients. Let $\theta$ be a pure state of $I$ to which $F, L, E_{0}$ are affiliated, as before. Then $E=E_{0} \cap \log f$ is a dominant stratum of $\log f$ (with respect to $F$ ) by II.3A. Clearly $f_{E}=f_{E_{0}}$. With $\psi: I \rightarrow \mathbb{R}$ defined as above via $E_{0}$ (so that $\psi=\theta$ ),

$$
\theta\left(\frac{f}{P}\right)=\theta\left(\frac{P^{m} f}{P^{m+1}}\right)=\psi\left(\frac{P^{m} f}{P^{m+1}}\right)=\theta\left(\frac{\left(P^{m} f\right)_{E_{0}+m F}}{P^{m+1}}\right)
$$

If $w$ belongs to $\log P^{m} f \cap\left(E_{0}+m F\right)$, writing $w=\sum w_{i}+v$ with $w_{i}$ in $\log P$ and $b$ in $\log f(\subset \log I)$, we have that the $w_{i}$ all belong to $F$ and $v$ lies in $E_{0} \cap \log f=E$ by the lemma above. Hence

$$
\left(P^{m} f\right)_{E_{0}+m F}=\left(P_{F}\right)^{m} f_{E_{0}}=\left(P_{F}\right)^{m} f_{E}
$$

of course $E$ is not empty by II.3.

Since for all $w$ in $E+m F$, say $w=e+\sum w_{i}$,

$$
\theta\left(\frac{x^{w}}{P^{m+1}}\right)=\theta\left(\frac{x^{e}}{P}\right) \cdot \prod L\left(\frac{x^{w_{i}}}{P}\right) \neq 0,
$$

we have that $\theta\left(x^{w} / P^{m+1}\right) \neq 0$. Thus

$$
\theta\left(\frac{\left(P^{m} f\right)_{E_{0}+m F}}{P^{m+1}}\right)>0 .
$$

So $f / P$ is strictly positive at all pure states of $I$, whence $f / P$ belongs to $R^{+}$, and we are done. 
Notice that we only use the dominant strata of $\log I$, begging the question as to whether a stratum sitting over a dominant stratum of $\log f$ is actually dominant (in $\log I$ ). This slight weakening of the hypothesis allows the induction process in the proof of theorem $B$ to take place.

\section{Converse of theorem A}

Let $E$ be a dominant stratum of $\log f$ with respect to the relative face $F$ of $\log P$. Suppose that $P^{n} f$ has only non-negative coefficients. We wish to show that the same is true of $\left(P_{F}\right)^{n} f_{E}$ (the same $n$ ).

We first observe that if $x$ belongs to $N F$ and $x=\sum w_{i}$ with $w_{i}$ in $\log P(i=$ $1,2, \ldots, N$ ), then the $w_{i}$ actually belong to $F$ (for example, $F$ is a dominant stratum of $\log P$ with respect to $F$ itself, and II.4 can be applied iteratively). Thus $\left(P^{n}\right)_{n F}=\left(P_{F}\right)^{n}$.

If $w=s+t$ appears in $\log P^{n} f$ with $s$ in $n \log P=\log P^{n}$, and $t$ in $\log f$, and if $w$ is a member of $n F+E$, then by II.4, $s \in n F$ and $t \in E$. It easily follows that

$$
\left(P^{n} f\right)_{n F+E}=\left(P^{n}\right)_{n F} \cdot f_{E}=\left(P_{F}\right)^{n} \cdot f_{E} \text {. }
$$

However, $P^{n} f$ has only non-negative coefficients, so that the same is true of $\left(P_{F}\right)^{n} f_{E^{*}}$

\section{Statement and proof of the second theorem}

THEOREM B. Suppose $f, P$ are elements of $\mathbb{R}\left[x_{i}^{ \pm 1}\right]$, with $P$ having only non-negative coefficients. There exists $n$ so that $P^{n} f$ has no negative coefficients if and only if the following conditions hold:

(a) As in the statement of theorem A, (a).

$\left(b^{\prime}\right)$ For each proper relative face $F$ of $\log P$, and each dominant stratum $E$ of $\log f$ (with respect of $F$ ),

$$
f_{E} \text { is strictly positive as a function on }\left(\mathbb{R}^{d}\right)^{++} \text {. }
$$

Since $f_{E}$ is not identically zero (by definition of $E$ ), condition (b) of theorem A implies $\left(b^{\prime}\right)$. We need only show that theorem $A$ may be applied iteratively (starting from the lower dimensional faces) to obtain that $\left(b^{\prime}\right)$ implies (b) (in the presence of (a)). We shall elaborate on this point later.

A definition is required, which formalizes two of the comments in $\S$ II.

Let $S$ be a subset of $\log P$, let $I_{S}$ be the ideal of $R$ generated by $\left\{x^{w} / P \mid w \in S\right\}$, and set $\log I_{S}=\left\{w \in \log P \mid x^{w} / P \in I_{s}\right\}$. (In our case, $S=\log f, I_{S}=I$.) A dominant stratum of $S$ is extendible if there exists a dominant stratum $E^{\prime}$ of $\log I_{S}$ such that $E^{\prime} \cap S=E$.

The proof of sufficiency in theorem A requires in place of (b) only:

$\left(b_{1}\right)$ For each proper relative face $F$, for each extendible dominant stratum $E$ of $\log f$ with respect to $F$, there exists an integer $m$ such that $P_{F}^{m} f_{E}$ has no negative coefficients.

(That is, 'extendible' has been inserted.)

Returning to the proof, let $E$ be an extendible dominant stratum of $\log f$, with respect to $F$. Suppose that $E \subset n F+z_{0}$. Set $g=x^{-z_{0}} f_{E}$, so that $\log g \subset E-z_{0} \subset n F$. Let $F^{\prime}$ be a relative face of $F$ (so $F^{\prime}$ is also a relative face of $\log P$ ); then $n F^{\prime}$ is 
a relative face of $n F$. Suppose that $K$ is an extendible dominant stratum of $\log g=$ $E-z_{0}$ with respect to $n F^{\prime}$, viewed as a relative face of $n F\left(=\log \left(P_{F}\right)^{n}=\log P_{F}^{n}=\right.$ $n \log P_{F}$ ). Observe that if $P$ is replaced by $Q=P_{F}^{n}$, then $n F=\log Q$; so we regard $\log g$ as a subset of $n F$, and consider its extendible dominant strata with respect to $n F^{\prime}$.

We shall show that $K+z_{0}$ is a dominant stratum of $\log f$ with respect to $F^{\prime}$ (viewed as a relative face of $\log P$ ). Before doing this, however, we briefly outline how theorem $B$ then follows from this result (more detail will be given at the end of the section). Apply theorem A with $g$ replacing $f$; since the affine dimension of (for example) the convex hull of $F$ will be smaller than that of $\operatorname{cvx} \log P$, an induction argument may be used.

That $K+z_{0}$ is a stratum of $\log f$ is routine: Obviously, $K+z_{0}$ is contained in a translate of an iterate of $F^{\prime}$. Suppose $K+z_{0} \subset N F^{\prime}+z$. We must show that $K+z_{0}=$ $\left(N F^{\prime}+z\right) \cap \log f$. Now

$$
K+z_{0} \subset\left(N F^{\prime}+z\right) \cap \log f \subset(N F+z) \cap \log f \subset E ;
$$

(the last inclusion follows from $K+z_{0} \subset E$, and $E$ being a stratum with respect to $F$, for $\log f)$. Select $w$ in $\log f \cap\left(N F^{\prime}+z\right)$. Since $w$ belongs to $E, w$ belongs to $\left(N F^{\prime}+z\right) \cap E=K+z_{0}$ (since $K$ is a stratum inside $E-z_{0}$ with respect to $F^{\prime}$ ), and this shows that $K+z_{0}$ is a stratum of $\log f$.

The difficult part is to show that $K+z_{0}$ is dominant, as a stratum of $\log f$. We observed in the reduction process of the first part of $\S$ II, that $E$ being a dominant stratum of a set $S$ with respect to the relative face $F$ of $\log P$ is requivalent to it being a dominant stratum with respect to the relative face $n F$ of $n \log P$. Hence we may assume $n=1$ (for notational convenience) here. Thus $E \subset F+z_{0}$, etc. The next results give an even more explicit description of the pure states of $I$, than that of $\S \mathrm{II}$.

Let $\not h$ be the ideal of $R$ generated by $\left\{x^{w} / P \mid w \in \log P \backslash F\right\}$. We saw earlier (just before II.1) that $h$ is an order ideal, and in fact as in [H; VII], it is a prime ideal and $R / h$ is order-isomorphic to $R_{P_{F}}$ that is, to $\mathbb{R}\left[x^{w} / P_{F} ; w \in F\right]$. Then point evaluations are faithful pure states there (that is, the kernels miss the positive cone). The map $R \rightarrow R_{P_{F}}$ with kernel $h$ is determined by the assignment (for $w$ in $\log P$ )

$$
x^{w} / P \mapsto \begin{cases}\frac{x^{w}}{P_{F}} & \text { if } w \in F, \\ 0 & \text { if } w \notin F .\end{cases}
$$

Let $L$ be a pure state on $R$ obtained by composing the map $R \rightarrow R_{P_{F}}$ with a faithful pure state of the latter. Then $\operatorname{ker} L \cap R^{+}=h^{+}$, so that for $w$ in $\log P, L\left(x^{w} / P\right)=0$ if and only if $w$ does not belong to $F$.

Say $E$ is a dominant stratum of $\log I$, with respect to $F$, a relative face of $\log P$. Say $L$ is a pure state as above, and $E \subset n F+z$ (this $n, z$ are not assumed to be $1, z_{0}$ respectively). For an element $a$ of $\mathbb{R}\left[x_{i}^{ \pm 1}\right]$ with $\log a \subset \log I+(k-1) \log P$ (for some $k$ ), set

$$
\theta\left(\frac{a}{P^{k}}\right)=L\left(\frac{x^{-z} a_{E+(k-1) F}}{P^{k+n-1}}\right) .
$$


Then $\theta$ is a well-defined real-valued function of $I$, and is a state; moreover, $E=$ $\left\{w \in \log I \mid \theta\left(x^{w} / P\right) \neq 0\right\}$, and furthermore, for all $s$ in $I$ and $r$ in $R, \theta(s r)=\theta(s) L(r)$.

Proof of well-definedness of $\theta$. This echoes the proof that $\psi$ (§II) is well defined. Suppose $a / P^{k}$ lies in $I$; we may assume that $\log a \subset \log _{k} I+(k-1) \log P$ (as in the corresponding proof for $\psi$ ). Suppose that $a / P^{k}=b / P^{m}$ with $m>k$ and $\log b \subset$ $\log I+(m-1) \log P$. Then $b=P^{m-k} a$. Setting

from $b=P^{m-k} a$, we deduce

$$
a=\sum \mu_{v} x^{v}, \quad b=\sum r_{w} x^{w}, \quad P^{m-k}=\sum_{(m-k) \log P} \lambda_{s} x^{s}
$$

$$
\sum r_{w} x^{w}=\sum_{w}\left(\sum_{\substack{s+v=w \\ s \in k \log P \\ v \in \log _{k} I}} \lambda_{s} \mu_{v}\right) x^{w}
$$

so

$$
b_{E+(m-1) F}=\sum_{w \in E+(m-1) F}\left(\sum_{\substack{s+v=w \\ s \in(m-k) \log P \\ v \in \log _{k} I}} \lambda_{s} \mu_{v}\right) x^{w} .
$$

By II.4, and that $E+(k-1) F$ is a dominant stratum of $\log _{k} I$, the ' $s \in(m-k) \log P$ ' condition may be replaced by ' $s \in(m-k) F$ ', and ' $v \in \log _{k} I$ ' may be replaced by ' $v \in E+(k-1) F$ '. The resulting expression for $b_{E+(m-1) F}$ is

$$
\left(\sum_{(m-k) F} \lambda_{s} x^{s}\right) \cdot\left(\sum_{E+(k-1) F} \mu_{v} x^{v}\right)
$$

which equals

$$
\left(P^{m-k}\right)_{(m-k) F} \cdot a_{E+(k-1) F} .
$$

For $w$ in $(m-k) \log P \backslash(m-k) F, L\left(x^{w} / P^{m-k}\right)=0$. Hence

$$
\begin{aligned}
L\left(\frac{b_{E+(m-1) F} x^{-z}}{P^{m+n-1}}\right) & =L\left(\left(\frac{\left(P^{m-k}\right)_{(m-k) F}}{P^{m-k}}\right) \cdot\left(\frac{x^{-z} a_{E+(k-1) F}}{P^{k+n-1}}\right)\right) \\
& =L\left(\frac{\left(P^{m-k}\right)_{(m-k) F}}{P^{m-k}}\right) L\left(\frac{x^{-z} a_{E+(k-1) F}}{P^{k+n-1}}\right) \\
& =1 \cdot L\left(\frac{x^{-z} a_{E+(k-1) F}}{P^{k+n-1}}\right),
\end{aligned}
$$

as desired. Thus $\theta$ is well defined.

Additivity is routine and is verified exactly as for $\psi$. For $w$ in $\log I+(k-1) \log P$, $\theta\left(x^{w} / P^{k}\right)=L\left(x^{w-z} / P^{k+n-1}\right) \geq 0$, so $\theta$ is positive (and thus a state of $\left.I\right)$. For $w$ in $\log I, \theta\left(x^{w} / P\right) \neq 0$ if and only if $w-z \in n F$, i.e. $w \in(n F+z) \cap \log I=E$. Finally, write $s=a / P^{k}$ ( $a$ as above), $r=c / P^{m}(\log c \subset m \log P)$. Then

$$
\begin{aligned}
\theta(s r)=\theta\left(a c / P^{m+k}\right) & \left.=L\left((a c)_{E+(m+k-1) F} x^{-z}\right) / P^{m+k}\right) \\
& =L\left(\frac{x^{-2} a_{E+(k-1) F}}{P^{k}}\right) \cdot L\left(\frac{c_{m F}}{P^{m}}\right) \\
& =\theta\left(\frac{a}{P^{k}}\right) \cdot L\left(\frac{c}{P^{m}}\right)
\end{aligned}
$$


(the latter, since $L$ annihilates elements of the form $x^{w} / P^{m}$ with $w$ in $m \log P \backslash m F$ ), as desired.

Corollary IV.1. A stratum $E$ of $\log I$ is dominant if and only if

implies

$$
E \cap((n \log P+z) \backslash(n F+z)) \neq \varnothing
$$

$$
(n F+z) \cap \log I=\varnothing .
$$

Proof. Certainly a stratum satisfying (*) is dominant by part (iii) of the definition. Conversely suppose that $E$ is a dominant stratum satisfying the first line of (*). Select $e$ in the intersection, so that $e=\sum w_{i}+z$, where the $w_{i}$ belong to $P$, but not all of them to $F$. Select is possible $w=\sum v_{j}+z$ in $\log I$ with $v_{j}$ in $F$. Then $w+\sum w_{i}=$ $e+\sum v_{j}$, so with $\theta$ as defined above applied,

$$
\theta\left(\frac{x^{w}}{P}\right) \prod L\left(\frac{x^{w_{i}}}{P}\right)=\theta\left(\frac{x^{e}}{P}\right) \prod L\left(\frac{x^{v_{i}}}{P}\right) .
$$

The left side is zero, since at least one of the $w_{i}$ is not in $F$. The right side is not zero, since $e$ belongs to $E$, and all of the $v_{j}$ 's lie in $F$. This contradicts the existence of such a $w$.

Corollary IV.1A. If $E_{0}$ is a dominant stratum of $\log I$ with respect to a relative face $F$ of $\log P$, then $E=\log f \cap E_{0}$ is a dominant stratum of $\log f$ with respect to $F$. Proof. Given $E_{0}$, there exists by the construction preceding IV.1, a pure state $\theta$ of $I$ such that $E_{0}=\left\{w \in \log I \mid \theta\left(x^{w} / P\right) \neq 0\right\}$. Since $E$ is thus $\left\{w \in \log f \mid \theta\left(x^{w} / P\right) \neq 0\right\}$, the result follows from II.3A.

Proposition IV.2. Let $S$ be a subset of $\log P$ and $F$ a relative face of $\log P$. If $E$ is an extendible dominant stratum of $S$ with respect to $F$, then $E$ satisfies:

$$
E \cap((n \log P+z) \backslash(n F+z)) \neq \varnothing
$$

implies

$$
(n F+z) \cap S=\varnothing .
$$

Proof. Extend $E$ to a dominant stratum $E^{\prime}$ of $\log I_{s^{*}}$ Then $E \cap$ $((n \log P+z) \cap(n F+z)) \neq \varnothing$ implies the same with $E^{\prime}$ in place of $E$. By IV.1, $(n F+z) \cap \log I_{S}=\varnothing$, and the result follows.

Finally we are in a position to show that if $K$ is an extendible dominant stratum of $\log g$ with respect to $F^{\prime}$, and $E$ is an extendible dominant stratum of $\log f$ with respect to $F$, then $K+z_{0}$ is a dominant stratum of $\log f$ with respect to $F^{\prime}$; in fact, we show that the formally stronger condition $(* *)$ of IV.2 holds.

Suppose that $\left(K+z_{0}\right) \cap\left((n \log P+z) \backslash\left(n F^{\prime}+z\right)\right) \neq \varnothing$. Select $w$ in $\log f \cap$ $\left(n F^{\prime}+z\right)$; we must show that the existence of $w$ leads to a contradiction. Since $E$ satisfies $(* *)$ (as a subset of $\log f$, and with respect to $F$ ), we must have that

$$
E \cap(n \log P+z) \subset n F+z .
$$

As $K+z_{0} \subset E,\left(K+z_{0}\right) \cap(n \log P+z) \subset n F+z$. Hence

$$
\left(K+z_{0}\right) \cap\left((n F+z) \backslash\left(n F^{\prime}+z\right)\right) \neq \varnothing .
$$


Since $K$ is an extendible dominant stratum of $\log g=E-z_{0}$ (within $F$ ) with respect to $F^{\prime}$, we have from

$$
K \cap\left(\left(n F+z-z_{0}\right) \backslash\left(n F^{\prime}+z-z_{0}\right)\right) \neq \varnothing
$$

that $\left(E-z_{0}\right) \cap\left(n F^{\prime}+z-z_{0}\right)=\varnothing$ (applying $(* *)$ to $\left.K\right)$, whence $E \cap\left(n F^{\prime}+z\right)=\varnothing$.

Since a subset of $E$ (namely $K+z_{0}$ ) lies in $n F+z$, it follows from I.1 that $\log f \cap(n F+z) \subset E$. Hence $w$ belongs to $E$, so $w$ belongs to $E \cap\left(n F^{\prime}+z\right)=\varnothing$, the desired contradiction.

Thus $K+z_{0}$ satisfies (**), and is thus a dominant stratum of $\log f$ as a subset of $\log P$, with respect to $F^{\prime}$.

Now the sufficiency of conditions $(a),\left(b^{\prime}\right)$ can be proved by induction on the affine dimension of the convex hull of the relative faces. If $F$ is zero dimensional (i.e. a single point), then the dominant strata are singletons, and the $f_{E}$ 's being positive as functions means that they are each of the form (positive real number) - (monomial), so that condition (b) of theorem $A$ holds in this case. Now assume that (b) holds for all relative faces $F^{\prime}$ of $\log P$ properly contained in $F$ (these necessarily have smaller affine dimension than $F$ ), and let $E$ be an extendible dominant stratum with respect to $F$, of $\log f$.

If we now replace $f$ by $g=x^{-z_{0}} f_{E}$, we see that theorem $\mathrm{A}$ applies to $g$, as follows. As ( $\left.\mathrm{b}^{\prime}\right)$ holds for $f,\left(\mathrm{~b}^{\prime}\right)$ holds for $f_{E}$ (because of the transitivity result for dominant substrata proved just above), hence for $g$. By induction (as the dimension of the convex hull of $F$ is strictly less than that of $\log P$ ), there exists a positive integer $m$ so that $\left(P_{F}\right)^{m} g$ has only non-negative coefficients, so that the same is true of $\left(P_{F}\right)^{m} f_{E}$. Now $f$ satisfies the hypotheses of theorem $\mathrm{A}$, and thus we may apply it, to obtain the existence of an integer $N$ with $P^{N} f$ having no negative coefficients.

\section{Examples}

We first study the case of one variable $(d=1)$.

Example V.1. To begin with, suppose that $P$ (or some power) is gap-free, meaning that if $x^{a}, x^{b}$ appear in $P$ and $a<c<b$ with $c$ an integer, than $x^{c}$ appears in $P$.

This case is easy, and the solution is already known (e.g. [H1; V.1]). The obvious necessary condition, $f \mid(0, \infty)>0$, is sufficient. To see this, by replacing $P$ by a power of itself, we may assume that $\log f \subset \log P$; it easily follows (as $P$ is gap-free) that the only dominant strata of $\log f$ are the singletons consisting of the endpoints, and their coefficients are automatically positive from the positivity of $f$ on $(0, \infty)$.

Example V.2. Now suppose that $P=x^{3}+x+1$ (or any of its relatives).

Here $\log P^{m}=\{0,1,2, \ldots, 3 m-2,3 m\}$; it has a gap, in that $3 m-1$ is missing. Multiplying $f$ by a suitable monomial, we may assume $\log f \subset\{0,1, \ldots, 3 m-2\}$, and that 0 belongs to $\log f$. Say $k=\operatorname{degree}(f)$ (so $k \leq 3 m-2$ ). Clearly $\{0\},\{k\}$ are dominant strata (for $F=\{0\}, F=\{3 m\}$ respectively). If $k-1$ belongs to $\log f$, then $\{k-1\}$ is a dominant stratum with respect to $F=\{3 m\}$, for if $k-1 \in$ $(n \log P+N) \backslash\{3 m n+N\}$, we have that $N \leq k-1 \leq 3 m n+N-2$, so $N+1 \leq k \leq$ $3 m n+N-1$; thus $(3 m n+N) \cap \log f$ is empty (as $\log f=\{0,1, \ldots, k\})$. Hence the coefficient of the second highest term in $f$ must be non-negative. 
It is straightforward to verify that no other dominant strata exist (in fact, with $n=1, \quad z=0)$. We may obviously replace $P$ by anything whose $\log$ set is $\{0,1,2, \ldots, t-2, t\}$, and we have the following.

Assume $P=\sum_{0}^{t} a_{i} x^{i}$ with $a_{i}>0$ except that $a_{t-1}=0$. Let $f=\sum_{0}^{k} b_{j} x^{j}$ with $b_{0}, b_{k}$ both not zero. Then there exists an integer $n$ so that $P^{n} f$ has no negative coefficients if and only if $f \mid(0, \infty)>0$ and $b_{k-1} \geq 0$.

Example V.3. Now try $P=x^{4}+x+1$ and its relatives. Here $\log P^{m}=$ $\{0,1, \ldots, 4 m\} \backslash\{4 m-5,4 m-2,4 m-1\}$ for $m \geq 2$. A similar analysis to that above shows that if $f=\sum_{0}^{k} b_{j} x^{j}\left(b_{0}, b_{k}\right.$ both not zero), then provided that $b_{k-5}, b_{k-2}$, and $b_{k-1}$ are (respectively) not zero, the corresponding singletons are the only additional dominant strata. Hence we deduce that $P^{n} f$ will have no negative coefficients for some $n$ if and only if $f \mid(0, \infty)>0$ and $b_{k-5}, b_{k-2}, b_{k-1}$ are all non-negative.

Both in this case and the previous ones, the necessity of these conditions is quite easy to obtain by direct computation, but sufficiency does not seem to have an elementary proof, except by either theorem A or B.

Example V.4. Now let $P=1+x^{2}+x^{5}$. Then $\log P=\{0,2,5\}$, and for $m \geq 4$, the gaps of $\log P^{m}$ are $\{1,3,5 m-7,5 m-4,5 m-2,5 m-1\}$. Hence if $f=\sum_{0}^{k} b_{j} x^{j}, b_{0}, b_{k}$ not zero, necessary and sufficient conditions that $P^{n} f$ be non-negative for some $n$ are that $f \mid(0, \infty)>0$ and that all of $b_{1}, b_{3}, b_{k-7}, b_{k-4}, b_{k-2}, b_{k-1}$ be non-negative.

So in the general one variable situation, reduce to a projectively faithful character (as in the reduction argument of $\S \mathrm{II}$; this was not necessary in any of the examples above, because the polynomials $P$ were already projectively faithful), determine the eventual gap pattern (and if $\operatorname{deg} P=t$, this occurs before the $t$ th power of $P$ ), and the criterion falls out.

Example V.5. We consider an example in two variables, $x$ and $y$. Suppose $P=$ $(1+x)(1+y)$, and $f=A x+B y+C x y^{2}+D x^{2} y^{3}+E x^{2} y+Z x^{2} y^{2}+M x y$, where the capital letters represent non-zero real numbers. Suppose additionally that $f$ is known to be strictly positive as a function on $\left(\mathbb{R}^{2}\right)^{++}$. We wish to determine conditions on the coefficients appearing in $f$, so that there will be an integer $n$ such that $P^{n} f$ has no negative terms.

Setting $S=\log f$, and replacing $P$ by $P^{3}$, this is exactly the situation described in figure 1 ( $\S \mathrm{I})$. The obvious (unions of) dominant strata corresponding to the faces of the convex hull of $\log f$ are:

$\{(1,0)\}$ : This yields $A>0$;

$\{(2,1)\}$ : so $E>0$;

$\{(2,3)\}$ : whence $D>0$;

$\{(0,1)\}$ : thus $B>0$;

$\{(0,1),(1,2),(2,3)\}$ : from which $y\left(B+C(x y)+D(x y)^{2}\right)$ is a strictly positive function in the single variable $x y$; in other words, either $C>0$ or $C^{2}>4 B D$ in the presence of the known inequalities $B>0$ and $D>0$;

$\{(2,1),(2,2),(2,3)\}$ : so $x^{2} y\left(E+Z y+D y^{2}\right)$ is strictly positive as a function, and thus either $Z>0$ or $Z^{2}<4 D E$;

and two others yielding no new information. 
There is an additional set of three dominant starta arising from the vertex $\{(0,3)\}$ (of $\log P^{3}$ ); each of $\{(0,1)\},\{(1,2)\},\{(2,3)\}$. We thereby deduce that $C>0$. All other dominant strata yield no new implications.

Hence, assuming $f$ is strictly positive on $\left(\mathbb{R}^{2}\right)^{++}$(only here does $M$ play any role), necessary and sufficient conditions are: $A, B, C, D, E>0$ and either $Z>0$ or $Z^{2}<$ $4 B D$.

In the following, we formalize one of the phenomena occurring in the last example.

LeMMA V.1. Let $\log f \subset \log P \subset \mathbb{Z}^{d}$, and let $D$ be a relative face of $\log f$ (including possibly $D=\log f$ ), such that the affine dimension of $\operatorname{cvx} D$ is strictly less than that of $\mathrm{cvx} \log P$. Then $D$ is a union of dominant strata of $\log f$, with respect to the same relative face of $\log P$. Moreover, if no line on the boundary of $\operatorname{cvx} \log P$ joining two vertices of $\log P$ is parallel to a line in the convex hull of $D$, then each point of $D$ constitutes a singleton dominant stratum.

Proof. Since $D$ is a relative face of $\log f$, there exists a linear functional $\alpha$ of $\mathbb{R}^{d}$ such that $\log f \subset\left\{r=\left(r_{i}\right) \in \mathbb{R}^{d} \mid \alpha(r) \leq 1\right\}$, and $D=\log f \cap\left\{r \in \mathbb{R}^{d} \mid \alpha(r)=1\right\}$ (the convex hull of $D$ is a face of the convex hull of $\log F)$. Let

$$
a=\sup \{\alpha(r) \mid r \in \operatorname{cvx} \log P\}
$$

necessarily, $a \geq 1$. Then $V=\left\{r \in \mathbb{R}^{d} \mid \alpha(r)=a\right\}$ is non-void and $V \cap \operatorname{cvx} \log P$ is a proper face of $\operatorname{cvx} \log P$, with $F=\log P \cap V$ being the corresponding relative face of $\log P$.

Select $d$ in $D$, and let $E$ be the stratum of $\log f$ with respect to $f$ that contains $d$. First we show that $E$ is contained in $D$, and then that $E$ is dominant. Say $E \subset(n F+z) \cap \log f$ for some integer $n$ and lattice point $z$, and select $e$ in $E$. Write $e=\sum w_{i}+z, d=\sum_{i=1}^{n} v_{i}+z$ with $v_{i}, w_{i}$ all belonging to $F$. Then $d+\sum w_{i}=e+\sum v_{i}$. Applying $\alpha$, and recalling that $\alpha(d)=1, \alpha\left(w_{i}\right)=\alpha\left(v_{i}\right)=a$, we deduce that $\alpha(e)=$ $\alpha(d)=1$, so $e$ belongs to $\log f \cap\{r \mid \alpha(r)=1\}=D$. Thus $E \subset D$.

Now suppose that $E \subset\left(m \log P+z_{0}\right) \backslash\left(m F+z_{0}\right)$ and that $\log f \cap\left(m F+z_{0}\right) \neq \varnothing$. Select $u$ in $\log f \cap\left(m F+z_{0}\right)$; write $u=\sum w_{i}+z_{0}$ with $w_{i}$ in $F$. Choose $e$ in $E$ with $e=\sum v_{i}+z_{0}$ where the $v_{i}$ belong to $\log P$, but not all of them lie in $F$. Applying $\alpha$ to $e$ and $u$, we see that

$$
\begin{aligned}
1=\alpha(e)=\sum \alpha\left(v_{i}\right)+\alpha\left(z_{0}\right) & <m a+\alpha\left(z_{0}\right) \\
& =\sum \alpha\left(w_{i}\right)+\alpha\left(z_{0}\right) \\
& =\alpha(u) .
\end{aligned}
$$

This yields $\alpha(u)>1$, a contradiction to $u$ belonging to $\log f$. So $E$ is a dominant stratum containing $d$ and contained in $D$. Thus $D$ is the union of the dominant strata that contain a point of $D$, all strata with respect to $F$.

If the additional hypothesis holds, then the $F$ constructed above must be a singleton (unless $D$ is itself a singleton), so all strata with respect to it must be singletons.

This lemma suggests that $P^{n} f$ having no negative coefficients for a specific $P$ is a very strong condition on $f$. For example, if $P=(1+x)(1+y)$ (so cvx $\log P$ is the 


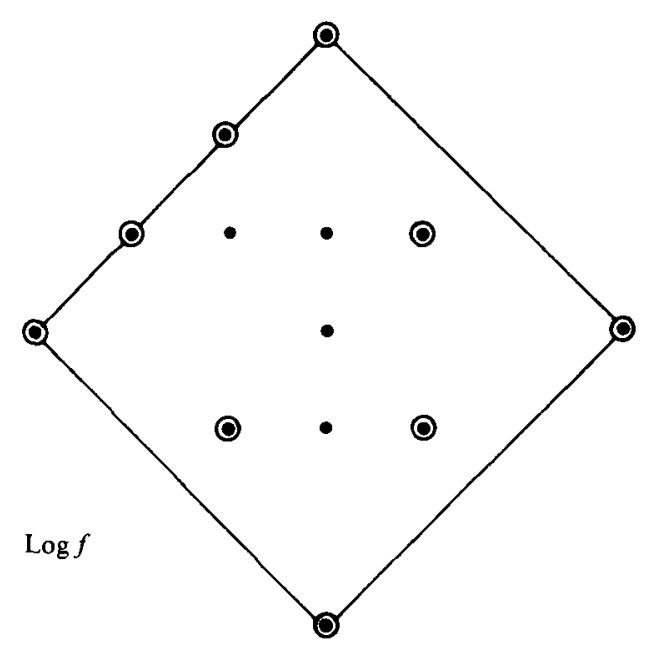

Figure 3. With respect to $P=(1+x)(1+y)$, the encircled points of $\log f$ correspond to monomials which must have positive coefficients, if there is to exist $n$ so that $P^{n} f$ has only non-negative coefficients.

unit square), and $\operatorname{cvx} \log f$ is a diamond shape, a necessary condition for there to exist $n$ such that $P^{n} f$ has no negative coefficients is that the coefficients of each of the monomials on the boundary of $\log f$ are positive. See figure 3 .

On the other hand, even when each relative face of $\log f$ is parallel to a relative face of $\log P$ (say for $d \geq 2$ ), more constraints intervene. A case in point is the following.

Example V.6. Set $P=1+x+y, f=1+x^{2}-x y+y^{2}$; then $\log f \subset \log P^{2}$, and with respect to $F=\{(2,0),(1,1),(0,2)\}$ (a relative face of $\log P^{2}$ ), the only dominant stratum is $E=F$.

Since $f_{E}=x^{2}-x y+y^{2}=x^{2}\left(1-\left(x^{-1} y\right)+\left(x^{-1} y\right)^{2}\right)$ is strictly positive, it follows from theorem B that $P^{n} f$ has no negative coefficients for large enough $n$. However, $P^{m} f_{E}$ always has a negative coefficient, since for $E=\log f_{E}$, the dominant strata with respect to $\{(0,0)\}$ (a face of $\log P^{2}$ ) are the three singletons $\{(2,0)\},\{(1,1)\},\{(0,2)\}$, and the coefficient corresponding to the second one is -1 .

A more general result is available.

Corollary V.2. Let $F$ be a relative face of $\log P$. Let $V$ be the affine span of all subfaces of $\operatorname{cvx} F$, of affine dimension at least one. Suppose that $\log f \subset V \cap \log P$ (e.g. $\log f \subset F$ ), and $\log P \not \subset V$. Then $P^{m} f$ having only non-negative coefficients for some integer $m$ implies that the coefficients of $f$ are already non-negative.

Proof. Set $D=\log f$, and apply the second statement of V.1. As $\log f$ decomposes into singleton dominant strata, by the converse to theorem $\mathrm{A}$, all the coefficients of $f$ must be non-negative.

Now suppose $P, P^{\prime}$ are polynomials in two variables with no negative coefficients, with both being solid (i.e. $\log P=\mathbb{Z}^{2} \cap \mathrm{cvx} \log P$ ), and the affine dimension of both cvx $\log P$, cvx Log $P^{\prime}$ being two (as opposed to one or zero). We wish to determine 
a relation (necessary and sufficient) between $\log P$ and $\log P^{\prime}$ so that for all polynomials $f$, the existence of $n$ so that $P^{n} f$ has only non-negative coefficients is equivalent to the existence of $m$ so that $\left(P^{\prime}\right)^{m} f$ has only non-negative coefficients. We shall say $P$ is positively equivalent to $P^{\prime}$ when this occurs. Mercifully, only an outline will be given of the proof that the relation described below determines exactly when $P$ and $P^{\prime}$ are positively equivalent.

Let $K, K^{\prime}$ denote cvx $\log P, \operatorname{cvx} \log P^{\prime}$ respectively. Since we are assuming both $P, P^{\prime}$ are solid, specifying (e.g.) $K$ is equivalent to specifying $\log P$. Extend each edge of $K$ (i.e. each one dimensional face of the convex polygon $K$ ) to a line. We can create a new convex polygon by parallel-transporting each of these lines (independently of each other), and taking the interior (if any). If $K^{\prime}$ has the same number of sides as $K$, and $K^{\prime}$ can be obtained by this process, then $P$ and $P^{\prime}$ are positively equivalent. Another way to describe this relation between $K$ and $K^{\prime}$ is the following.

Label the sides of $K, s_{0}, s_{1}, \ldots, s_{k}$ so that the $i+1$ st side $(\bmod k+1)$ is the 'next' side to the $i$ th one, going in a counter-clockwise direction $\left(s_{i+1}\right.$ is adjacent to $s_{i}$, and the angle between them measured from $s_{i}$ and proceeding in a clockwise direction is less than $180^{\circ}$ ). Similarly label the sides of $K^{\prime}, s_{0}^{\prime}, s_{1}^{\prime}, \ldots, s_{k^{\prime}}^{\prime}$. Then $P$ and $P^{\prime}$ will be positively equivalent if and only if $k=k^{\prime}$ and there is a cyclic permutation $\pi$ of $(0,1, \ldots, k)$ so that each $s_{i}$ is parallel to $s_{\pi(i)}^{\prime}$.

To see this, first suppose one face of $K^{\prime}$, say $s_{0}^{\prime}$, is not parallel to any $s_{i}$. Replace $P^{\prime}$ by $\left(P^{\prime}\right)^{2}$ to guarantee that $s_{0}^{\prime} \cap \log P^{\prime}$ has at least three points (as opposed to the two obvious ones arising from the endpoints, which are vertices of $\log P^{\prime}$ ). Define $f$ a polynomial, with $\log f=d_{e} \log P^{\prime} \cup\{e\}$, where $e$ is a point in in $s_{0}^{\prime} \cap \log P^{\prime}$ that is not a vertex. Let the coefficients be 1 for all the monomials corresponding to vertices, and -1 for $x^{e}$. By observing that the polynomial in one variable (which is a translate of $f_{E}$, where $E$ is the three-element set consisting of the two endpoints of $s_{0}^{\prime}$ and $\left.e\right) X^{k}-X^{m}+1(k>m)$ is always strictly positive, we see immediately from theorem $B$, that there exists a power of $P^{\prime}$ such that $\left(P^{\prime}\right)^{n} f$ has only non-negative coefficients. On the other hand, since $s_{0}^{\prime}$ is not parallel to any face of $\log P$, it follows from V.1 that $E$ decomposes into three singleton dominant strata relative to some relative face of $\log P$, and since the coefficient of $x^{e}$ is -1 , for no $n$ has $P^{n} f$ only non-negative coefficients.

So every face of $K$ is parallel to a face of $K^{\prime}$, and conversely.

Now suppose $s_{0}, s_{1}, \ldots, s_{j}$ are parallel respectively to $s_{0}^{\prime}, \ldots, s_{j}^{\prime}$, and moreover, $s_{0}$ is not parallel to any other $s_{i}$ (this leaves out the case of a $2 n$-gon, where every side is parallel to its opposite-this has to be treated separately). If $s_{j+1}$ is not parallel to $s_{j+1}^{\prime}$, then essentially the same procedure as above constructs an $f$. That is, we may assume that $s_{j+1}^{\prime} \cap \log P^{\prime}$ contains at least three points, define the polynomial $f$ to have coefficient one at each of the vertices' monomials, and -1 at a non-vertex occurring in $s_{j+1}^{\prime} \cap \log P^{\prime}$. Then relative to the relative face $s_{j+1} \cap \log P, E$ decomposes into three singleton dominant strata as above, and the same argument works.

There remains the special case, where every face of $K$ is parallel to exactly one other face. Here the problem is that there are two possible faces $s_{0}^{\prime}$ to start with 
and both must be taken into consideration, but essentially the same argument as above works for both.

To prove the converse is trivial-the dominant strata of $f$ with respect to a relative face $F$ of $\log P$ are identical to those with respect to $\pi(F)$ ( $\pi$ acts on the vertices as well), and theorem $B$ applies.

The author was supported in part by an operating grant from NSERC of Canada.

\section{REFERENCES}

[EHS] E. G. Effros, D. E. Handelman, \& C.-L. Shen. Dimension groups and their affine representations. Amer. J. Math. 102 (1980), 385-407.

[H1] D. Handelman. Positive polynomials and product type actions of compact groups. Mem. Amer. Math. Soc. 320 (1985) 79p+xii.

[HR] D. Handelman \& W. Rossmann. Non-product type actions of compact groups on AF algebras. Illinois J. Math. 29 (1985), 51-95. 